\title{
Minireview \\ Nuclear EGFR signalling network in cancers: linking EGFR pathway to cell cycle progression, nitric oxide pathway and patient survival
}

\author{
H-W Lo' and M-C Hung*,I \\ 'Department of Molecular and Cellular Oncology, University of Texas MD Anderson Cancer Center, 1515 Holcombe Blvd, Houston, TX 77030, USA
}

\begin{abstract}
Emerging evidences suggest the existence of a new mode of epidermal growth factor receptor (EGFR) signalling pathway in which activated EGFR undergoes nuclear translocalization and subsequently regulates gene expression and potentially mediates other cellular processes. This signalling route is distinct from the better-characterized, traditional EGFR pathway that involves transduction of mitogenic signals through activation of multiple signalling cascades. Transcriptional activity of nuclear EGFR appears to depend on its C-terminal transactivation domain and its physical and functional interaction with other transcription factors that contain DNAbinding activity. Likely via its ability to upregulate gene expression, nuclear EGFR pathway is associated with major characteristics of more aggressive tumours: increased proliferative potential, nitric oxide synthesis, and accelerated GI/S cell cycle progression. A role of nuclear EGFR in prognostic prediction is further suggested in patients with breast carcinomas and oropharyngeal squamous cell carcinomas. It is noted that significant advances were made towards the knowledge of the nuclear EGFR pathway; however, many aspects of this new pathway remain unresolved and will be discussed in this review. As a number of other receptor tyrosine kinases (RTKs) and cytokine receptors also undergo similar nuclear translocalization, a better understanding of the physiological and malignant nature of the nuclear EGFR pathway will likely shed light into the biology of cancer with nuclear RTKs.

British Journal of Cancer (2006) 94, I84- I88. doi: I0.1038/sj.bjc.660294 I www.bjcancer.com
\end{abstract}

(c) 2006 Cancer Research UK

Keywords: epidermal growth factor receptor; inducible nitric oxide synthase; transcriptional regulation; cell cycle control; receptor tyrosine kinase

Albeit very little is known about physiological function and cancer relevance of the nuclear EGFR pathway until recent years, EGFR has been consistently detected in the nuclei of cancer cells and primary tumour specimens of various origins as well as in those of other highly proliferative tissues (Marti et al, 1991; Cao et al, 1995; Lin et al, 2001; Lo et al, 2005a, b). Increased expression of nuclear EGFR is linked to poor clinical outcome in patients with breast carcinomas (Lo et $a l, 2005 \mathrm{c}$ ) and oropharyngeal squamous cell carcinomas (Psyrri et al, 2005). Consistently, nuclear accumulation of EGFR correlates with increased expression of cyclin D1, inducible nitric oxide synthase (iNOS) and B-Myb, all of which are frequently overexpressed in human cancers and associated with increased cell proliferation (Lin et al, 2001; Hanada et al, 2005; Lo et al, 2005a). Furthermore, most recent reports indicate a plausible mechanism underlying nuclear EGFR-mediated gene regulation, which involves a physical interaction with other transcription factors, signal transducer and activator of transcription 3 (STAT3) and E2F1 (Hanada et al, 2005; Lo et al, 2005a). A cellular mechanism that can potentially account for nuclear import of receptor tyrosine kinases (RTKs) is also proposed recently (Giri et al, 2005). It is important to note that nuclear import is not only observed with EGFR but also with many other RTKs, including mouse erbb1, HER-2, rat p185neu, HER-3, truncated C-terminal HER-4, and fibroblast growth factor receptor (FGFR) and cytokine

*Correspondence: Dr M-C Hung; E-mail: mhung@mdanderson.org Received 4 November 2005; revised 5 December 2005; accepted 6 December 2005 receptors (Marti et al, 1991; Xie and Hung, 1994; Lin et al, 2001; Ni et al, 2001; Offterdinger et al, 2002; Schausberger et al, 2003; Wang et al, 2004; Krolewski, 2005). As RTK-mediated pathways are frequently deregulated in many human cancers and are closely linked to tumorigenesis and tumour progression, it is thus an urgent task to better understand the nature of these nuclear RTKs and, more importantly, to determine the extent to which they contribute to the malignant biology and therapeutic response of human cancers.

\section{DETECTION OF NUCLEAR EGFR}

Nuclear detection of EGFR was first reported in hepatocytes that underwent regeneration and in primary adrenocortical carcinomas more than a decade ago (Kamio et al, 1990; Marti et al, 1991). Nuclear expression of EGFR was further detected in other cell types and tissues, such as placentas, thyroids and immortalized epithelial cells of ovary and kidney origins (Cao et al, 1995; Lin et al, 2001; Marti et al, 2001; Lo et al, 2005a). High levels of EGFR was also found in the nuclei of many tumours, including those of skin, breast, bladder, cervix, adrenocorticord, thyroid and oral cavity (Kamio et al, 1990; Lipponen and Eskelinen, 1994; Lin et al, 2001; Marti et al, 2001; Lo et al, 2005a, c; Psyrri et al, 2005). In addition, EGFR has been shown to localize in the inner nuclear membrane (Cao et al, 1995; Klein et al, 2004). Nuclear counterpart of EGFR appears to be the full-length receptor and likely, in the phosphorylated form, as shown by a number of studies (Cao et al, 1995; Lin et al, 2001; Cordero et al, 2002; Dittmann et al, 2005a; 
Lo et al, 2005a, c). Consistently, EGF and pro-TGF- $\alpha$ were found to translocate into the nucleus of proliferating hepatocytes (Raper et al, 1987; Schausberger et al, 2003). In addition to ligand stimulation, nuclear translocalization of EGFR can be initiated by irradiation, heat shock, $\mathrm{H}_{2} \mathrm{O}_{2}$ and cisplatin (Cao et al, 1995; Lin et al, 2001; Dittmann et al, 2005a). Conversely, EGF- and irradiation-induced EGFR nuclear transport can be blocked by 1,25-dihydroxyvitamin D (Cordero et al, 2002) and anti-EGFR antibody, C225/Cetuximab (Dittmann et al, 2005b), respectively.

\section{NUCLEAR EXISTENCE OF OTHER RTKS}

Nuclear translocalization is not a unique event with EGFR but rather a universal phenomenon occurring to many other cellsurface receptors. In addition to EGFR and its mouse homologue erbb1, all other receptors in the ErbB family have also been detected in the nucleus (Xie and Hung, 1994; Lin et al, 2001; Ni et al, 2001; Offterdinger et al, 2002; Schausberger et al, 2003; Wang et al, 2004; Krolewski, 2005). Rat p185neu, HER-2 and HER-3 receptors exist as full-length receptors in cell nucleus (Xie and Hung, 1994; Offterdinger et al, 2002; Wang et al, 2004) whereas HER-4 undergoes $\gamma$-secretase-mediated cleavage and the C- terminal $80-\mathrm{kDa}$ fragment translocates into the nucleus (Ni et al, 2001). Several other RTKs, including TrkA,B/NGFR, FGFR, VEGF receptor 2 (VEGFR-2) and type I TGF- $\beta$ receptor also undergo nuclear transport (Rakowicz-Szulczynska et al, 1988; Maher, 1996; Zwaagstra et al, 2000; Pillai et al, 2005). Inflammatory cytokine receptors, such as, those of interleukin-1 (IL-1), IL-5 and interferon- $\gamma$ (IFN- $\gamma$ ) also exist in the nuclear compartment (Curtis et al, 1990; Jans and Hassan, 1998; Larkin et al, 2000). In line with these observations, ligands to most of these receptors were also found in the nucleus (Raper et al, 1987; Curtis et al, 1990; Jans and Hassan, 1998; Schausberger et al, 2003).

\section{POTENTIAL MECHANISMS FOR NUCLEAR - CYTOPLASMIC TRANSPORT OF CELL-SURFACE RECEPTORS}

Several lines of evidences suggest that many nuclear RTKs can be originated from the cell-surface counterparts and exist as uncleaved full-length receptors, including EGFR, HER-2, HER-3, and FGFR1 (Maher, 1996; Lin et al, 2001; Offterdinger et al, 2002; Wang et al, 2004; Dittmann et al, 2005a; Giri et al, 2005; Lo et al, 2005c) (Figure 1). VEGFR2 (Shay-Salit et al, 2002), IFN $\gamma \mathrm{R}-1, \mathrm{IFN} \gamma \mathrm{R}-2$

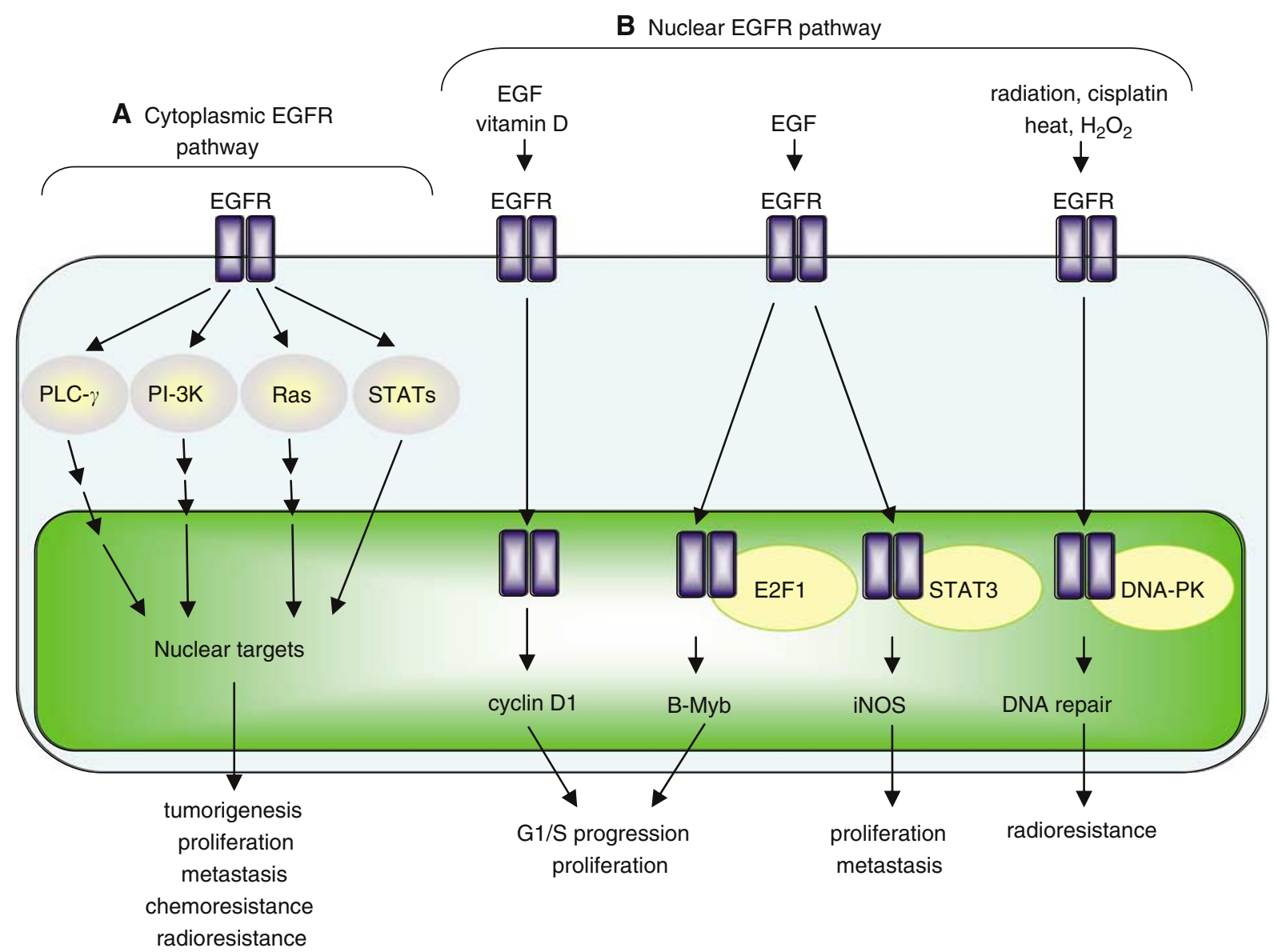

Figure I Cytoplasmic/traditional and nuclear modes of the EGFR signalling pathway. The EGFR signalling pathway exerts its biological effects via two major modes of actions, namely, cytoplasmic/traditional $(\mathbf{A})$ and nuclear $(\mathbf{B})$ modes. (A) The cytoplasmic EGFR pathway is consisted of four major modules: PLC- $\gamma$-CaMKJPKC, Ras-Raf-MAPK, PI-3K-Akt-GSK and STATs. Activation of these signalling modules often leads to tumorigenesis, tumour proliferation, metastasis, chemoresistance and radioresistance. (B) The nuclear EGFR pathway can be initiated by ligand binding and exposure to vitamin D, radiation, cisplatin, heat and $\mathrm{H}_{2} \mathrm{O}_{2}$. Following nuclear translocalization, nuclear EGFR interacts with DNA-binding transcription factors, E2FI and STAT3, and activates expression of B-Myb and iNOS, respectively. Nuclear EGFR also upregulates cyclin DI gene expression. Increased expression of cyclin DI and B-Myb contributes to accelerated GI/S cell cycle progression and, on the other hand, elevated iNOS is associated with tumour proliferation and metastasis. Upon DNA damage and oxidative/heat stress, EGFR enters the cell nucleus and interacts with DNA-PK, leading to DNA repair and radioresistance. 
(Jans and Hassan, 1998; Larkin et al, 2000), IL-1R (Curtis et al, 1990) also localize in the nucleus as intact receptors. Spliced variants of cell-surface FGFR1 and the intracellular domain of HER-4 have been found to enter the cell nucleus (Stachowiak et al, 1997; Ni et al, 2001, 2003); however, it is yet clear whether TrkA, TrkB (Zhang et al, 2003), type I TGF- $\beta$ receptor (Zwaagstra et al, 2000) and IL-5R (Jans et al, 1997) undergo nuclear translocalization as full-length receptors. While the mechanisms underlying nuclear import of the intracellular domain of HER-4 are bettercharacterized (Ni et al, 2001, 2003), those account for full-length receptors remain less clear as they are complicated by the membrane-associated nature of the full-length receptors.

Nuclear import of cell-surface receptors has been shown to occur in ligand-dependent and -independent manners. Ligand stimulation activates nuclear translocalization of many cell-surface receptors including EGFR, FGFR, IFN- $\gamma$ R, IL- $1 \mathrm{R}$, type I TGF- $\beta$ receptor and IL-5R (Curtis et al, 1990; Jans and Hassan, 1998; Larkin et al, 2000; Zwaagstra et al, 2000; Lin et al, 2001). In the case of HER-2 which lacks the domain/ability for ligand-binding, its kinase activity is required for nuclear entry (Wang et al, 2004). Nuclear import of EGFR and type I TGF- $\beta$ receptor can occur in a ligand-independent manner (Zwaagstra et al, 2000; Dittmann et al, 2005a). In contrast, heregulin $\beta 1$ activates nuclear export of HER-3 in mammary epithelial cells, which contain high levels of nuclear HER-3 under normal growth conditions (Offterdinger et al, 2002).

For those cell-surface receptors that undergo nuclear import following ligand binding, it is speculated and supported by several recent reports that receptor internalization may serve as an initial step for its transit from the cell-surface to the nucleus, as ligand activation is coupled with receptor internalization (Bryant and Stow, 2005). Blocking receptor internalization/endocytosis using Dynamin II mutant, Dynamin II K44A, prevents nuclear import of EGFR, HER-2 and FGFR (Bryant et al, 2005; Giri et al, 2005; Lo et al). However, it is yet clear with regard to the individual contribution of clathrin-, lipid raft- and caveolin-dependent endocytosis to this process. Several lines of evidences further suggest the possibility that endocytic sorting machinery may be utilized to shuttle internalized receptors to the perinuclear and nuclear regions (Bryant et al, 2005; Giri et al, 2005).

In the light of the observation that many cell-surface receptors with nuclear-cytoplasmic shuttling appear to exist as nonmembrane-bound receptors in the nucleus, it is thus speculated that cells may utilize a general mechanism for extracting these transmembrane receptors prior to its passage through the nuclear pore complex (NPC). However, such mechanism has not yet identified. Nevertheless, it is becoming clear that several cellsurface receptors are capable of interacting with nuclear transport receptors, importins $\alpha / \beta$ and exportins, and thus enter and exit the cell nucleus, respectively (Reilly and Maher, 2001; Giri et al, 2005). Association with importin $\beta 1$ is important for nuclear import of HER-2 and FGFR (Reilly and Maher, 2001; Giri et al, 2005) whereas EGFR has been shown to interacts with both importin $\beta 1$ and importin $\alpha$ (Dittmann et al, 2005a; Lo et al). Blockage of RanGDP and importin $\beta 1$ by dominant-negative mutant and siRNA, respectively, also inhibits nuclear transport of HER-2, suggesting that NPC is involved in the process (Giri et al, 2005).

Nuclear localization signals (NLSs) within EGFR, HER-2, HER-3 and HER-4 have been identified (Offterdinger et al, 2002; Wang et al, 2004; Williams et al, 2004; Lo et al, 2005a). Interestingly, EGFR, HER-2 and HER-4 contain their NLSs within the juxtamembrane region whereas HER- $3^{\prime}$ s NLS is located in the C-terminal region. On the other hand, very little is known about the nuclear export process. Recent evidences suggest that nuclear export of EGFR, HER-2 and HER-3 may involve exportin CRM1 (Offterdinger et al, 2002; Giri et al, 2005; Lo et al). Existence of nuclear export sequences within these cell-surface receptors, however, has not been demonstrated.

\section{NUCLEAR EGFR AND HER-2 AS TRANSCRIPTIONAL REGULATOR}

A role of nuclear ErbBs in transcriptional regulation was first shown by the observation that cytoplasmic domain of rat p185neu contains transactivational activity when tested in a GAL4-reporter system (Xie and Hung, 1994). More recently, transactivational domains within EGFR, HER-2 and HER-4 were also found to be functional (Lin et al, 2001; Ni et al, 2001; Wang et al, 2004). Using unbiased approaches, nuclear EGFR and HER-2 were further shown to associate with specific DNA sequences designated ATrich sequence (ATRS) and HER-2-associated sequence (HAS), respectively (Lin et al, 2001; Wang et al, 2004). Promoters that are targeted by nuclear EGFR are those of cyclin D1, iNOS and B-Myb (Lin et al, 2001; Hanada et al, 2005; Lo et al, 2005a). Nuclear HER-2 binds to promoters of cyclooxygenase-2 (COX-2), PRPK, MMP-16 and DDX-10, whereas nuclear HER-4 associates with that of $\beta$-casein (Wang et al, 2004; Williams et al, 2004).

Given the notion that ErbB receptors lack a putative DNAbinding domain, it is suspected that these receptors first associate with DNA-binding transcription factors and then enhance target gene transcription via their intrinsic transactivational activity. In this regard, nuclear EGFR interacts with STAT3 and coregulates iNOS expression (Lo et al, 2005a). Nuclear EGFR/E2F1 complex activates expression of B-Myb, a positive regulator of $\mathrm{G} 1 / \mathrm{S}$ cell cycle progression (Hanada et al, 2005). Nuclear HER-4 forms a complex with STAT5a and coactivates $\beta$-casein gene promoter (Williams et al, 2004). However, it is suggested that HER-4 may have a weak transactivational activity and requires interaction with a strong transcription coactivator YAP for its gene regulatory function (Komuro et al, 2003). In line with this observation, nuclear FGFR associates with and activates transcription coactivator CBP to upregulate gene promoters (Fang et al, 2005). Known targets of nuclear FGFR include FGF-2, neurofilament-L and tyrosine hydroxylase (Peng et al, 2001, 2002; Stachowiak et al, 2003). In support of this notion, EGF, EGFR, TrkA/NGF receptor and the ligand NGF have been shown to bind to chromatins (Rakowicz-Szulczynska et al, 1988; Kamio et al, 1990) and Schwannoma-derived growth factor, a ligand for EGFR, bound to A + T-rich DNA sequences (Kimura, 1993). Together, these data suggest an emerging role that nuclear receptors play in transcriptional regulation.

\section{LINKING NUCLEAR EGFR TO PATHWAYS THAT ARE IMPORTANT FOR TUMOUR BIOLOGY}

Transcriptional targets of nuclear EGFR and HER-2, those identified thus far, are closely involved in tumorigenesis, and tumour proliferation and progression (Lin et al, 2001; Wang et al, 2004; Hanada et al, 2005; Lo et al, 2005a). Both cyclin D1 and BMyb are positive regulators of G1/S progression (Lin et al, 2001; Joaquin and Watson, 2003). COX-2 and iNOS are enzymes that produce prostagladins and nitric oxide, respectively, and emerge as major targets for chemoprevention and chemotherapy (Gupta and Dubois, 2001; Xu et al, 2002). Consistently, a positive correlation has been found between nuclear EGFR and cyclin D1/iNOS in a cohort of breast carcinomas (Lo et al, 2005a, c). In the same tumour cohort, high levels of nuclear HER-2 associate with COX-2 overexpression (Wang et al, 2004). Furthermore, nuclear expression of EGFR positively correlates with that of Ki-67, an indicator of active proliferation (Lo et al, 2005c). A casual correlation of nuclear accumulation of EGFR/mouse erbb1 and their ligands EGF/TGF- $\alpha$ with cell proliferation/DNA synthesis has been reported by several studies (Marti et al, 1991; Schausberger et al, 2003). In agreement with its role in proliferation/DNA synthesis, EGFR undergoes nuclear translocalization in regenerating livers (Marti et al, 1991), pregnant uterus and proliferative basal cells within normal mouth mucosa (Lin et al, 2001). A 
potential role of nuclear EGFR in DNA damage/repair in response to irradiation/oxidative stress has also been suggested (Dittmann et al, 2005a, b).

Furthermore, nuclear import of FGFR is associated with proliferation (Reilly and Maher, 2001), which is in line with the observation that nuclear FGFR enhances c-jun expression (Reilly and Maher, 2001). Also suggested by accumulating evidences is that nuclear FGFR mediates cAMP-activated expression of neurofilament-L and, thus, is important for cAMP-induced differentiation of neuronal progenitor cells into neurons (Stachowiak et al, 2003).

While the pathological significance of nuclear RTKs remains elusive, two recent reports suggest the potential use of nuclear EGFR as a prognostic indicator for poor clinical outcome (Lo et al, 2005c; Psyrri et al, 2005). In a cohort of 130 breast carcinomas, an inverse correlation was found between nuclear EGFR, but not the non-nuclear counterpart, and overall patient survival (Lo et al, 2005c). This observation is consistent with the notion that total EGFR levels serve as a moderate prognostic indicator in breast cancer patients and thus suggests a novel prognostic value of nuclear EGFR in these patients. A positive association was also observed between non-nuclear/nuclear EGFR and overall survival of patients with oral squamous cell carcinomas (Lo et al, 2005c). Using a cohort of 95 patients with oropharyngeal squamous cell carcinomas, Psyrri et al (2005) found that both total and nuclear EGFR levels predict poor clinical outcomes as measured by local recurrence and poor disease-free survival. However, no significant correlation was found between EGFR, total and nuclear expression, and overall survival rates in these patients (Psyrri et al, 2005). Despite with relatively small cohorts, these studies provided rationales for future extensive research that examines the prognostic value of EGFR in larger population with various cancer types. This task is particularly important and of cancer-relevance because of the following reasons: (i) the EGFR signalling pathway is highly de-regulated in many human cancers and is an attractive target for anti-tumour therapy, (ii) anti-EGFR therapy are only effective to certain patients, (iii) a correlation is often lacking between EGFR expression and tumour responsiveness to anti-EGFR treatments, and (iv) previously overlooked nuclear EGFR pathway is linked to aggressive tumour biology.

Moreover, in the light of the report showing that 1,25dihydroxyvitamin D inhibited EGF-induced EGFR nuclear transport, vitamin D1 may in part exert its anticancer effect via blocking the nuclear EGFR pathway (Cordero et al, 2002). Similarly, antiEGFR antibody (C225/Cetuximab) blocks radiation-induced nuclear EGFR transport and its interaction with DNA-dependent protein kinase, an enzyme involved in DNA-repair, and thus may lead to radiosensitization (Dittmann et al, 2005a, b). Interestingly, in women with breast cancer, nuclear HER-4 has been recently shown to associate with poor survival compared to those who had membrane HER-4 expression (Junttila et al, 2005). Together, these reports suggest that nuclear EGFR may play a potential important role in the aggressive biology of cancers, radiosensitivity and clinical outcomes.

\section{CONCLUSIONS}

Accumulating reports reveal a new EGFR signalling pathway that escapes the traditional transduction cascades but involves direct shuttling of activated EGFR into the cell nucleus. Nuclear existence of EGFR has been observed for more than a decade in normal cells undergoing active proliferation and in cancerous cells. The physiological function of nuclear EGFR, however, was not elucidated until recently to involve regulation of gene transcription and possibly other nuclear events. Nuclear EGFR, HER-2, HER-4 and FGFR contain intrinsic ability to enhance gene transcription. In addition to transcriptional regulation, nuclear RTKs may have other functions such as DNA damage/repair. Mechanistic studies further provide plausible mechanisms by which nuclear EGFR/ HER-4 turn on gene expression, which involve their intrinsic transactivation domain and physical interaction with other transcription factors that contain DNA-binding domains. STAT3 and E2F1 have been identified as transcription co-factors of nuclear EGFR whereas STAT5a partners with nuclear HER-4. Via the ability of nuclear EGFR to associate with STAT3 and E2F1 and to upregulate expression of cyclin D1, iNOS and B-Myb, a new link is established that associates EGFR with several cellular processes such as cell cycle progression and nitric oxide pathway. Consistent with the role in liver regeneration, nuclear EGFR accumulation is associated with increased proliferation in cancer cells and hepatocytes. Albeit correlative studies suggest an inverse association of high nuclear EGFR and poor clinical outcome, the pathological role of nuclear EGFR remains largely unknown and is in need of further investigations. For instance, it is yet to be determined whether nuclear EGFR plays a crucial role in the genesis, progression, metastatic growth and/or therapeutic responses of human cancers. Also elusive is the pathological involvement of nuclear HER-2, HER-3 and HER-4 in human cancers. All these unaddressed issues will be crucial in advancing our knowledge of the malignant nature of the nuclear RTK pathways. In summary, emerging new evidences provide important insights into a signalling path that has been overlooked for past decades and thus prompt for an urgent need to further unravel the physiological and oncogenic properties of the nuclear EGFR pathway.

\section{ACKNOWLEDGEMENTS}

This study is partially supported by RO-1 CA109311, PO-1 CA99031, the P50 83639, P20 CA101936 and MD Anderson Cancer Center, Cancer Center Supporting Grant, CA16672 (to M-CH) from the National Institute of Health. H-WL is a recipient of the Post-doctoral Fellowships from the American Cancer Society (PF-05-175-01-TBE to H-WL).

\section{REFERENCES}

Bryant DM, Stow JL (2005) Nuclear translocation of cell-surface receptors: lessons from fibroblast growth factor. Traffic 6: 947-954

Bryant DM, Wylie FG, Stow JL (2005) Regulation of endocytosis, nuclear translocation, and signaling of fibroblast growth factor receptor 1 by E-cadherin. Mol Biol Cell 16: 14-23

Cao H, Lei ZM, Bian L, Rao CV (1995) Functional nuclear epidermal growth factor receptors in human choriocarcinoma JEG-3 cells and normal human placenta. Endocrinology 136: 3163-3172

Cordero JB, Cozzolino M, Lu Y, Vidal M, Slatopolsky E, Stahl PD, Barbieri MA, Dusso A (2002) 1, 25-Dihydroxyvitamin D downregulates cell membrane growth- and nuclear growth-promoting signals by the epidermal growth factor receptor. J Biol Chem 277: $38965-38971$

Curtis BM, Widmer MB, deRoos P, Qwarnstrom EE (1990) IL-1 and its receptor are translocated to the nucleus. J Immunol 144: 1295-1303

Dittmann K, Mayer C, Fehrenbacher B, Schaller M, Raju U, Milas L, Chen DJ, Kehlbach R, Rodemann HP (2005a) Radiation-induced epidermal growth factor receptor nuclear import is linked to activation of DNAdependent protein kinase. J Biol Chem 280: $31182-31189$

Dittmann K, Mayer C, Rodemann HP (2005b) Inhibition of radiationinduced EGFR nuclear import by C225 (Cetuximab) suppresses DNA-PK activity. Radiother Oncol 76: $157-161$ 
Fang X, Stachowiak EK, Dunham-Ems SM, Klejbor I, Stachowiak MK (2005) Control of CREB-binding protein signaling by nuclear fibroblast growth factor receptor-1: a novel mechanism of gene regulation. $J$ Biol Chem 280: $28451-28462$

Giri DK, Ali-Seyed M, Li L-Y, Lee D-F, Ling P, Bartholomeusz G, Wang S-C, Hung M-C (2005) Endosomal Transport of ErbB-2: mechanism for nuclear entry of the cell surface receptor. Mol Cell Biol 25: 11005-11018

Gupta RA, Dubois RN (2001) Colorectal cancer prevention and treatment by inhibition of cyclooxygenase-2. Nat Rev Cancer 1: 11-21

Hanada N, Lo HW, Day CP, Pan Y, Nakajima Y, Hung MC (2005) Coregulation of B-Myb expression by E2F1 and EGF receptor. Mol Carcinog 45: $10-17$

Jans DA, Briggs LJ, Gustin SE, Jans P, Ford S, Young IG (1997) The cytokine interleukin-5 (IL-5) effects cotransport of its receptor subunits to the nucleus in vitro. FEBS Lett 410: $368-372$

Jans DA, Hassan G (1998) Nuclear targeting by growth factors, cytokines, and their receptors: a role in signaling? Bioessays 20: $400-411$

Joaquin M, Watson RJ (2003) Cell cycle regulation by the B-Myb transcription factor. Cell Mol Life Sci 60: 2389-2401

Junttila TT, Sundvall M, Lundin M, Lundin J, Tanner M, Harkonen P, Joensuu H, Isola J, Elenius K (2005) Cleavable ErbB4 isoform in estrogen receptor-regulated growth of breast cancer cells. Cancer Res 65: $1384-1393$

Kamio T, Shigematsu K, Sou H, Kawai K, Tsuchiyama H (1990) Immunohistochemical expression of epidermal growth factor receptors in human adrenocortical carcinoma. Hum Pathol 21: 277-282

Kimura H (1993) Schwannoma-derived growth factor must be transported into the nucleus to exert its mitogenic activity. Proc Natl Acad Sci USA 90: $2165-2169$

Klein C, Gensburger C, Freyermuth S, Nair BC, Labourdette G, Malviya AN (2004) A $120 \mathrm{kDa}$ nuclear phospholipase Cgammal protein fragment is stimulated in vivo by EGF signal phosphorylating nuclear membrane EGFR. Biochemistry 43: $15873-15883$

Komuro A, Nagai M, Navin NE, Sudol M (2003) WW domain-containing protein YAP associates with ErbB-4 and acts as a co-transcriptional activator for the carboxyl-terminal fragment of ErbB-4 that translocates to the nucleus. J Biol Chem 278: $33334-33341$

Krolewski JJ (2005) Cytokine and growth factor receptors in the nucleus: What's up with that? I Cell Biochem 95: 478-487

Larkin III J, Johnson HM, Subramaniam PS (2000) Differential nuclear localization of the IFNGR-1 and IFNGR-2 subunits of the IFN-gamma receptor complex following activation by IFN-gamma. J Interferon Cytokine Res 20: $565-576$

Lin SY, Makino K, Xia W, Matin A, Wen Y, Kwong KY, Bourguignon L, Hung MC (2001) Nuclear localization of EGF receptor and its potential new role as a transcription factor. Nat Cell Biol 3: 802-808

Lipponen P, Eskelinen M (1994) Expression of epidermal growth factor receptor in bladder cancer as related to established prognostic factors, oncoprotein (c-erbB-2, p53) expression and long-term prognosis. $\mathrm{Br} \mathrm{J}$ Cancer 69: 1120-1125

Lo H-W, Ali-Seyed M, Bartholomeusz G, Wu Y, Hung M-C. Nuclearcytoplasmic transport of EGFR involves receptor endocytosis importin $\beta$ 1 CRM1. J Cell Biochem, under review

Lo H-W, Hsu S-C, Ali-Seyed M, Gunduz M, Xia W, Wei Y, Bartholomeusz G, Shih J-Y, Hung M-C (2005a) Nuclear interaction of EGFR and STAT3 in the activation of iNOS/NO pathway. Cancer Cell 7: $575-589$

Lo HW, Hsu SC, Hung MC (2005b) EGFR signaling pathway in breast cancers: from traditional signal transduction to direct nuclear translocalization. Breast Cancer Res Treat 1-8; E-pub ahead of print

Lo H-W, Xia W, Wei Y, Ali-Seyed M, Huang SF, Hung M-C (2005c) Nove prognostic value of nuclear EGF receptor in breast cancer. Cancer Res 65: $338-348$

Maher PA (1996) Nuclear translocation of fibroblast growth factor (FGF) receptors in response to FGF-2. J Cell Biol 134: 529-536

Marti U, Burwen SJ, Wells A, Barker ME, Huling S, Feren AM, Jones AL (1991) Localization of epidermal growth factor receptor in hepatocyte nuclei. Hepatology 13: $15-20$

Marti U, Ruchti C, Kampf J, Thomas GA, Williams ED, Peter HJ, Gerber H, Burgi U (2001) Nuclear localization of epidermal growth factor and epidermal growth factor receptors in human thyroid tissues. Thyroid 11: $137-145$
Ni CY, Murphy MP, Golde TE, Carpenter G (2001) Gamma-secretase cleavage and nuclear localization of ErbB-4 receptor tyrosine kinase. Science 294: 2179-2181

Ni CY, Yuan H, Carpenter G (2003) Role of the ErbB-4 carboxyl terminus in gamma-secretase cleavage. J Biol Chem 278: $4561-4565$

Offterdinger M, Schofer C, Weipoltshammer K, Grunt TW (2002) c-erbB-3: a nuclear protein in mammary epithelial cells. J Cell Biol 157: 929-939

Peng H, Moffett J, Myers J, Fang X, Stachowiak EK, Maher P, Kratz E, Hines J, Fluharty SJ, Mizukoshi E, Bloom DC, Stachowiak MK (2001) Novel nuclear signaling pathway mediates activation of fibroblast growth factor- 2 gene by type 1 and type 2 angiotensin II receptors. Mol Biol Cell 12: $449-462$

Peng H, Myers J, Fang X, Stachowiak EK, Maher PA, Martins GG, Popescu G, Berezney R, Stachowiak MK (2002) Integrative nuclear FGFR1 signaling (INFS) pathway mediates activation of the tyrosine hydroxylase gene by angiotensin II, depolarization and protein kinase C. J Neurochem 81: $506-524$

Pillai G, Cook N, Turley H, Leek RD, Blasquez C, Pezzella F, Harris AL, Gatter KC (2005) The expression and cellular localization of phosphorylated VEGFR2 in lymphoma and non-neoplastic lymphadenopathy: an immunohistochemical study. Histopathology 46: 209-216

Psyrri A, Yu Z, Weinberger PM, Sasaki C, Haffty B, Camp R, Rimm D, Burtness BA (2005) Quantitative determination of nuclear and cytoplasmic epidermal growth factor receptor expression in oropharyngeal squamous cell cancer by using automated quantitative analysis. Clin Cancer Res 11: $5856-5862$

Rakowicz-Szulczynska EM, Herlyn M, Koprowski H (1988) Nerve growth factor receptors in chromatin of melanoma cells, proliferating melanocytes, and colorectal carcinoma cells in vitro. Cancer Res 48: 7200-7206

Raper SE, Burwen SJ, Barker ME, Jones AL (1987) Translocation of epidermal growth factor to the hepatocyte nucleus during rat liver regeneration. Gastroenterology 92: $1243-1250$

Reilly JF, Maher PA (2001) Importin beta-mediated nuclear import of fibroblast growth factor receptor: role in cell proliferation. J Cell Biol 152: $1307-1312$

Schausberger E, Eferl R, Parzefall W, Chabicovsky M, Breit P, Wagner EF, Schulte-Hermann R, Grasl-Kraupp B, Chabikovsky M (2003) Induction of DNA synthesis in primary mouse hepatocytes is associated with nuclear pro-transforming growth factor alpha and erbb-1 and is independent of c-jun. Carcinogenesis 24: $835-841$

Shay-Salit A, Shushy M, Wolfovitz E, Yahav H, Breviario F, Dejana E, Resnick N (2002) VEGF receptor 2 and the adherens junction as a mechanical transducer in vascular endothelial cells. Proc Natl Acad Sci USA 99: 9462 - 9467

Stachowiak EK, Fang X, Myers J, Dunham S, Stachowiak MK (2003) cAMPinduced differentiation of human neuronal progenitor cells is mediated by nuclear fibroblast growth factor receptor-1 (FGFR1). J Neurochem 84: $1296-1312$

Stachowiak EK, Maher PA, Tucholski J, Mordechai E, Joy A, Moffett J, Coons S, Stachowiak MK (1997) Nuclear accumulation of fibroblast growth factor receptors in human glial cells - association with cell proliferation. Oncogene 14: 2201-2211

Wang SC, Lien HC, Xia W, Chen IF, Lo HW, Wang Z, Ali-Seyed M, Lee DF, Bartholomeusz G, Ou-Yang F, Giri DK, Hung MC (2004) Binding at and transactivation of the COX-2 promoter by nuclear tyrosine kinase receptor ErbB-2. Cancer Cell 6: 251-261

Williams CC, Allison JG, Vidal GA, Burow ME, Beckman BS, Marrero L, Jones FE (2004) The ERBB4/HER4 receptor tyrosine kinase regulates gene expression by functioning as a STAT5A nuclear chaperone. J Cell Biol 167: $469-478$

Xie Y, Hung MC (1994) Nuclear localization of p185neu tyrosine kinase and its association with transcriptional transactivation. Biochem Biophys Res Commun 203: $1589-1598$

Xu W, Liu LZ, Loizidou M, Ahmed M, Charles IG (2002) The role of nitric oxide in cancer. Cell Res 12: $311-320$

Zhang FX, Lai CH, Lai SK, Yung KK, Shum DK, Chan YS (2003) Neurotrophin receptor immunostaining in the vestibular nuclei of rats. Neuroreport 14: $851-855$

Zwaagstra JC, Guimond A, O'Connor-McCourt MD (2000) Predominant intracellular localization of the type I transforming growth factor-beta receptor and increased nuclear accumulation after growth arrest. Exp Cell Res 258: $121-134$ 